\title{
Proposal of an Augmented Reality Tag UAV Positioning System for Power Line Tower Inspection
}

\author{
Alvaro Rogério Cantieri ${ }^{1(\bowtie)}$, Marco Aurélio Wehrmeister ${ }^{2}$, \\ André Schneider Oliveira ${ }^{2}$, José Lima ${ }^{3}$, Matheus Ferraz ${ }^{2}$, and Guido Szekir ${ }^{2}$ \\ 1 Federal Institute of Paraná, Curitiba, Paraná, Brazil \\ alvaro.cantieri@ifpr .edu.br \\ 2 Universidade Tecnológica Federal do Paraná, Curitiba, Paraná, Brazil \\ $\{$ wehrmeister, andreoliveira\}@utfpr.edu.br, \\ matf.ferraz@gmail.com, guidoszekir@gmail.com \\ 3 CeDRI - Research Centre in Digitalization and Intelligent Robotics, \\ Polytechnic Institute of Bragança and INESC TEC, Bragança, Portugal \\ jllima@ipb.pt \\ http://www.ifpr.edu.br, http://www.utfpr.edu.br/, \\ http://portal3.ipb.pt/index.php/pt/ipb
}

\begin{abstract}
Autonomous inspection Unmanned Aerial Vehicle systems are an essential research area, including power line distribution inspection. Considerable efforts to solve the demanding presented in the autonomous UAV inspection process are present in technical and scientific research. One of these challenges is the precise positioning and fly control of the UAV around the energy structures, which is vital to assure the security of the operation. The most common techniques to achieve precise positioning in UAV fly are Global Positioning Systems with RealTime Kinematic. This technique demands a proper satellite signal receiving to work appropriately, sometimes hard to achieve. The present work proposes a complementary position data system based on augmented reality tags (AR Tags) to increase the reliability of the UAV fly positioning system. The system application is proposed for energy power tower inspections as an example of use. The adaptation to other inspection tasks is possible whit some small changes. Experimental results have shown that an increase in the position accuracy is accomplished with the use of this schema.
\end{abstract}

Keywords: Power line inspection UAV - Autonomous UAV inspection $\cdot$ AR Tag UAV position

\section{Introduction}

The power line inspection process is a mandatory task made periodically by the energy distribution enterprises around the world. It is essential to maintain the 
security of the energy system and avoid energy delivery interruptions. Manual inspection process of power distribution towers is made by human operators that climb the structures to visualize details of the components. This kind of task is demanding and risky to the operator. Manual inspection is being replaced by the use of small-size unmanned aircraft vehicles equipped with image cameras and other sensors, which increases the security and efficacy of the process.

Several enterprises around the world execute inspection services based on UAV. Commonly, the aircraft is remotely controlled by a human pilot, that is responsible for control the navigation around the tower to achieve the necessary visual information of the components. An operation technician that works side by side to the pilot watch the video stream transmitted by the UAV and search for possible fails. The pilot is responsible for precise positioning and collision avoidance during the fly, which brings some restrictions to the flying route and tower proximity, for safety reasons.

An UAV capable of autonomous missions brings security and efficacy to the inspection process. Maintaining the position of the aircraft at the defined points and routes is one of the challenges present on these systems. A possible solution is the use of a Real-Time Kinematic GPS (RTK-GPS) system embedded on the aircraft. RTK-GPS is a technique that calculates the phase difference of two GPS signals received by separate modules and uses it to improve the output data accuracy to centimeter-level. This equipment provides excellent performance, and allows the creation of high secure autonomous position systems for the UAV's. Real-world situations affect the performance of RTK-GPS, like the small number of visible satellites, coverage of the receptor by environment structures, high trees, rain clouds, or other interference elements. When these situations occur, the centimeter-level accuracy is lost, and the navigation may become insecure.

This paper proposes the use of a position measurement methodology using a group of Augmented Reality Tag (AR-TAG), displaced around the tower on the ground. A down-pointing camera embedded on the UAV capture images of the tags, and a ROS package calculate it's position relative to the aircraft. This information to provide a secondary position data to the flight control algorithm.

A set of real-world experiments was executed, to evaluate the accuracy level of the position reading during the fly, measure the influence of environmental conditions, tag dimension, outdoor illumination, camera vibration, tag position and alignment among others.

A simulated experiment was proposed to evaluate the control capability of aircraft using the tags as reference, using the Virtual Robot Experimentation Platform (V-REP) software. The simulated system architecture is based on Robot Operating System (ROS) [11]. An overview of the proposed system is shown in Fig. 1. 


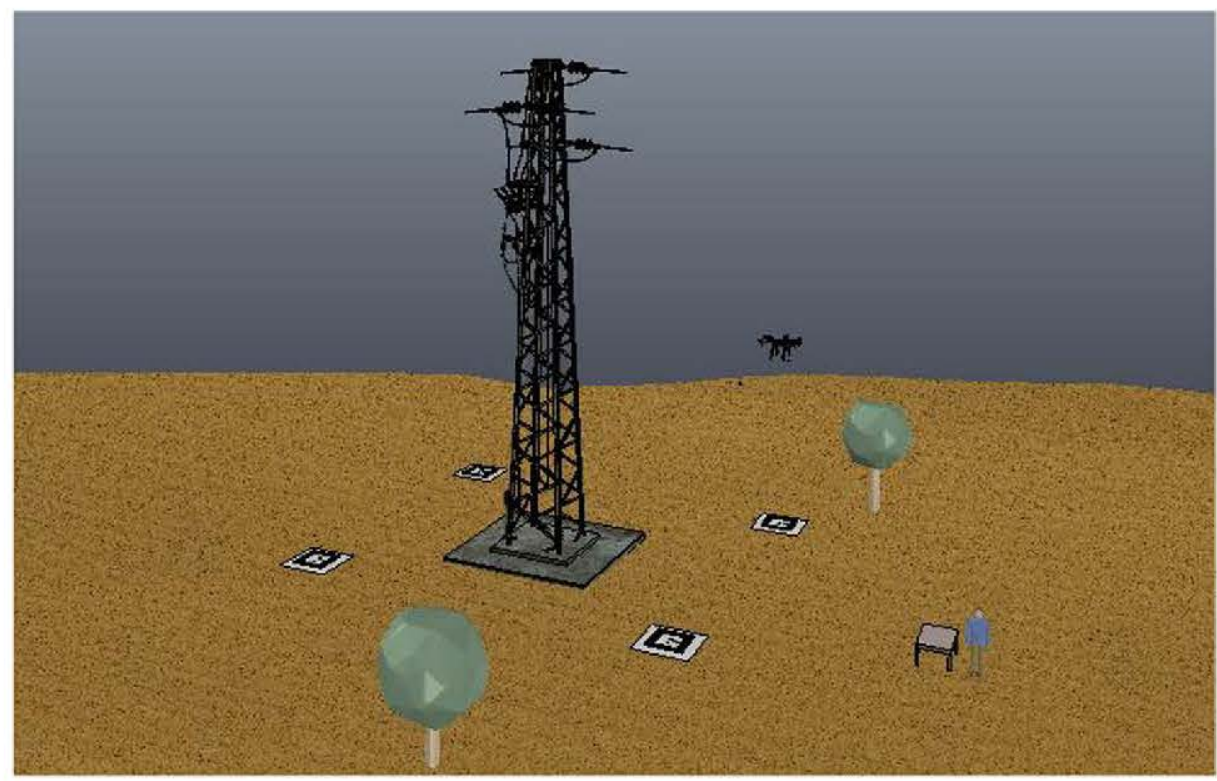

Fig. 1. Overview of the system

\section{Background and Related Works}

UAV power line inspection had grown in number in the last decade. The use of small size remote piloted UAV brings efficacy and security to the process, dispensing the necessity of a human to climb the structures to make the task.

A common way to execute autonomous UAV outdoor fly is to use a Global Navigation Satellite System (GNSS) position system. This technology offers a cheap and straightforward solution to navigate autonomous aircraft, compatible with the most of flight control hardware available on the market. The use of GNSS system to perform precise positioning to an UAV is not recommendable, because of the system accuracy. North America GPS for example, assure a 4-m accuracy performance, as described on [12].

A possible solution to increase the position accuracy of GPS data is the use of RTK -GPS. This technique executes calculations of the phase difference between two GPS signals received in different points, allowing to increase the position accuracy to centimeter-level. This technique demands a communication link between the base and the rover that receives and calculates the position corrections. The system also needs to receive a minimum number of satellite signals with adequate signal to noise rate to work correctly. The presence of obstacles like buildings, trees, clouds, and communication lost between the modules can cause the decrease of data accuracy level [13].

Some commercial UAV's offers RTK capabilities with autonomous fly. Small RTK modules for drone applications are also available on the global market. This technique is the best choice for precise positioning for UAV, but the problems described justify the development of a new approach for the position problem. 
The first UAV autonomous power line inspection systems presented in the literature uses GNSS position system. The proposal's focus is to make the UAV follow a path near to the transmission lines and send images of the structure to a base station. As an example of these systems, the work [10] describes the build and test of two small aircraft, a fix-wing and a tricopter. Both aircraft are controlled trough radio link, and executes flight missions using GPS positioning. These missions consist of autonomous following of a path near to the power line structures, to acquire images of their components.

The work [7] describes a quadrotor helicopter capable of autonomous path following using GPS for power line inspection apply. A regular camera and a thermal infra-red camera capture images of the structure and sends it to the base station. The aircraft performs three flight modes, manual flight, manually GPS assisted attitude fly an autonomous fly.

The propositions described above do not offer high precision positioning to detailed power line inspections. Complementary positioning systems must be added to the aircraft to achieve the position accuracy necessary to perform this task. Position algorithms based on computer vision systems are a possible approach to improve the position accuracy of a UAV autonomous fly. The base of this technique is to acquire and process environment images and identify elements and visual clues, estimating the position of the object relative to the UAV for each new image frame. Artificial intelligence and other techniques are applied to identify the reference points and calculate distances and feed the UAV fly control algorithms.

The work [6] use a stereoscopic image processing to calculate the UAV position related to the tower and power lines. The stereoscopic calculation algorithm uses two consecutive images to evaluate the position of an obstacle. The paper does not presents detailed information about the accuracy of the system to be compared whit other similar propositions.

The work [1] proposes a "Point-Line-Based SLAM" technique to provide a center tower position based on image processing. The system uses two highperformance GPU hardware embedded on the UAV to visual information processing. The results present an average position error of $0.72 \mathrm{~m}$. The disadvantage of the technique is the hardware demanding to process the visual information.

Processing images to calculate objects position is a complex task, which demands a lot of hardware capacity because of the high number of variables to be considered in this calculation. The use of Artificial Tags displaced on the environment to position calculation is an excellent approach to decrease the computational demanding of image positioning systems. This technique uses algorithms that process the visual information of specific draws printed, commonly called tags, disposed in known positions. The tags can provide 3D position, orientation, and even additional high-level information like numbers, strings, URL, etc, demanding low computational cost compared with the traditional image processing systems. 
Some tags positioning system described in the literature, originally proposed to Augmented Reality applications, has been applied to provide mobile robotic position and orientation data. April Tags [5] and AR Track Alvar [3] are example of these tools. The build of outdoor navigation systems using AR-Tags is a challenging task. Many environment parameters, like sunlight, tag distance, and visual obstacles that shadows the tag, impose difficulties in the correct processing of the tag images. Some recent published works provide background to assure the viability of using the artificial tags to offer outdoor position data to UAV fly.

The work [9] describes an outdoor UAV navigation system where the aircraft follows a moving automobile and executes an autonomous landing, using a group of AR-Tag placed in the automobile roof to get position and orientation data. The proposed system has success in perform correct positioning to the landing even when only using the visual position information provided by the tag.

The paper [4] proposes a UAV vision-based target positioning solution. The work develops a position control algorithm to find a ground target, represented by an and follow it. The authors argue that the solution provides $92 \%$ reliability in real-world experiments, showing the viability of using this kind of proposal to UAV outdoor positioning. The paper suggest the application of the solution in autonomous infrastructure inspection system developments, but do not test it in real inspection applications.

In this work, we propose a complementary position solution to apply in UAV autonomous power line detailed inspections, based in AR-Tags placed around the tower to serve as visual reference to the aircraft. The main contribution of this works is to investigate the viability of using AR-Tags in the proposed environmental and technical demands provided by the inspection problem. The second contribution is to provide a first approach solution to the autonomous inspection system, tested through the simulation environment.

\section{Architecture Specification}

Power line tower inspection is a complex task that demands a carefully visual data collection of several components of the structure and also near area. This project proposes an approach to the single tower inspection problem, a detailed process where the operator needs to visualize components of the tower, like insulators, spacers, dampers, conductors, fixing elements, etc. This visualization demands that the UAV reach some specific positions around the tower, and stay static while the camera is acquiring images of the point of interest.

This work is based on information exchange between the research group and the local energy distribution enterprise. It was essential to define clearly the operational parameters of the UAV inspection system proposition, like secure distance between the tower and the UAV, tower height, and navigation velocity during the inspection. A first approach was defined to validate the concept, using small size energy towers. Some operational parameters were chosen based on this definition, shown on Table 1. 
Table 1. Project parameter values defined to the system

\begin{tabular}{l|l}
\hline Parameter & Value \\
\hline Ideal distance between UAV tower during inspection & $4 \mathrm{~m}$ \\
\hline Minimal security distance between the UAV and the tower & $2 \mathrm{~m}$ \\
\hline Horizontal range of Tags visualization during the fly & $0 \mathrm{~m}-7 \mathrm{~m}$ \\
\hline Vertical range of Tags visualization during the fly & $10 \mathrm{~m}-40 \mathrm{~m}$ \\
\hline Maxim height of the inspected tower & $30 \mathrm{~m}$ \\
\hline Maxim UAV velocity of displacement during inspection & $0.5 \mathrm{~m} / \mathrm{s}$ \\
\hline Range of UAV inspection around the tower & $360^{\circ}$ \\
\hline
\end{tabular}

\subsection{System Components Description}

The system is composed of an UAV, a base station and four Augmented Reality tags disposed on the ground around power line tower, as shown in Fig. 1. The UAV camera captures the Tags image and sends it to the base station computer that runs the relative position calculation and transmits this data to the aircraft controller. Simulation and outdoor experiments were executed to evaluate the performance of architecture. The components and tools used to build the solution are described next.

Robot Operating System: System communication is made through Robot Operating System nodes, where the base station computer works as ROS Master [11]. ROS Kinetic runs on a PC that works as a base station, running Ubuntu 16.4 LTS. Computer system is a core i7 processor with 16 GB RAM and Intel® HD Graphics 520 (Skylake GT2) board.

Bebop Drone: A Bebop Drone quadcopter running ROS Bebop Autonomy driver was used to evaluate the position accuracy obtained by the proposed solution. Bebop drone is an excellent choice for this kind of test because it offers stabilized fly performance and embedded Full HD resolution camera gimbal stabilized, that minimizes the image displacement during the data acquisition. ROS Bebop Autonomy driver [8] is another advantage of this model, which makes it easy to exchange data between the drone and the base station during those experiments.

Ar Track Alvar Solution: The solution uses Ar-Track Alvar Augmented Reality [3] to implement the reference AR Tag system on this work. It provides flexible usage and excellent computational performance. The solution also allows multi-marker utilization, an advantage to the proposed architecture presented in this paper. The Ar Track Alvar package developed by Scott Niekum offers ROS compatibility. The Images of the AR-TAG are achieved by Bebop camera and processed by this package, that publishes position and orientation data on ar_pose_marker Ros Node. 
V-REP Simulation Environment: The simulated environment was created on V-REP software to evaluate the proposed YUAV autonomous navigation system performance. V-REP is a flexible and robust robotic simulation platform that offers a set of programming tools and robot component models, making it easy to test algorithms and robotic systems. An hexacopter model with PID position control script, four AR-Tags images displaced on the ground, a down-pointing camera embedded on the hexacopter frame and a power line tower model composes the environment. A ROS master execute all information exchanges between the components.

\subsection{Simulation Description}

Some modules compose the system, performing specific tasks on the simulation. All the simulation architecture system runs using ROS interface. The components exchange information between ROS to execute their tasks.

The hexacopter camera captures the AR Tag images and sends them to the package witch processes the calculation of the position of the tag, publishing this information in /visualization_marker ROS topic.

$\mathrm{A} \mathrm{C}++$ code receives this information and filter the position of the nearest tag visualized by the camera. The code performs a reference transformation and publishes the results in /position and /orientation ROS topic.

Another $\mathrm{C}++$ code publishes a group of waypoints on a /mission topic, one at time, to provide the position and orientation points that must be reached by the hexacopter in a mission simulation. Each point is published with a fixed time interval between the last, to assure that the hexacopoter have adequate time to make the displacement between then.

A script written in LUA language runs in V-REP simulator, implementing a PID position controller to the hexacopter. This PID code receives the position calculation and the next waypoint from the $\mathrm{C}++$ codes and performs the velocity calculations to displace the hexacopter to the desired point. This code is simple, and if the position provided is not correct, like when any tag is visible, the control of the aircraft is lost. Figure 2 shows an overview of the ROS topics and nodes running on this system.

\section{Experimental Methodology and System Evaluation Tests}

The architecture proposal followed methodological steps to guide the system conception. First, outside measurements using a 1-m size tag was run to evaluate the feasibility of the scheme. After that, a simulated environment based on the collected data, to test a control algorithm of the UAV using AR Tag readings. The experiment descriptions and results are shown next. 


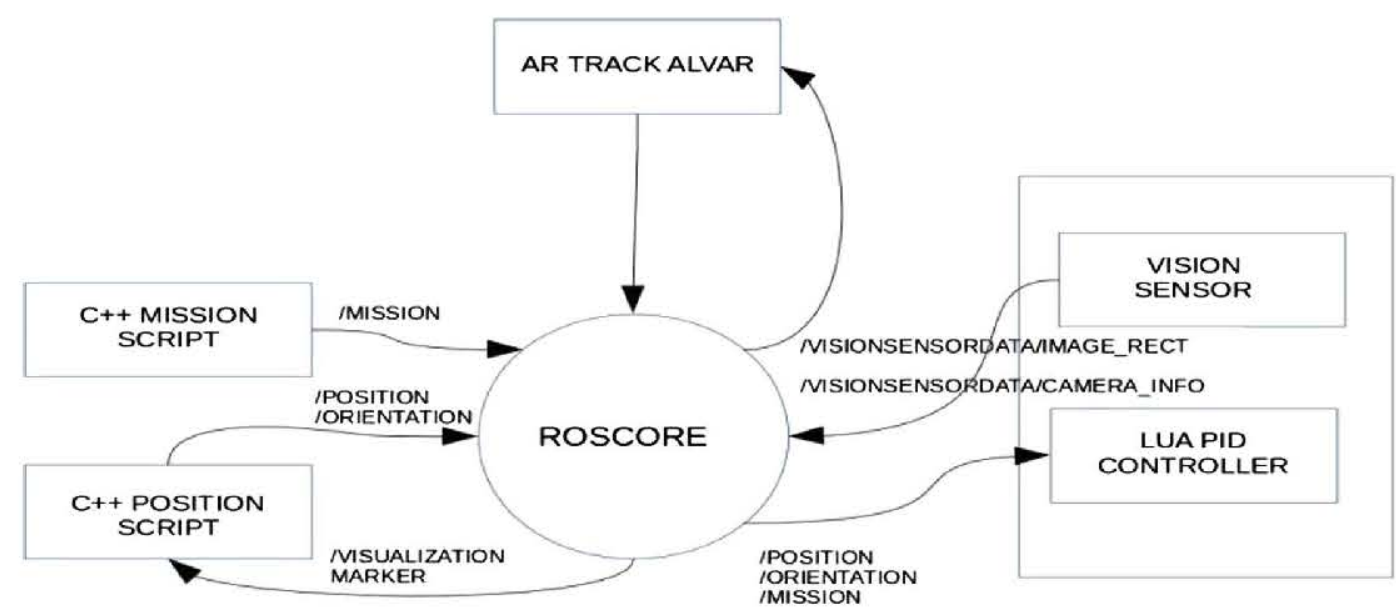

Fig. 2. ROS architecture of the simulation

\subsection{Position Reading by the UAV Using AR Tag in an Outdoor Environment}

A position accuracy evaluation provided by the AR Tag in an outdoor environment was executed using Bebop drone in a static position. The objective of this experiment is to evaluate if the position readings of the Bebop camera could provide adequate data between the distance range necessary to the system $(0 \mathrm{~m}$ to $40 \mathrm{~m}$ ).

The experiment uses a 1.0-m side size printed tag, placed in an outdoor space on a wall, with a $50 \mathrm{~m}$ measurement tape reference fixed on the ground. The tag is placed in front of Bebop drone with direct sunlight incidence. Bebop drone was placed on a desk, pointing the camera directly to the tag. The alignment of the drone was made using two wires fixed on the side of the tags and stretched over the entire length of the measuring shaft. The measurements were made in 4.0 $\mathrm{m}$ intervals, from 4.0 to $40.0 \mathrm{~m}$ distance. Four hundred position measurements samples of $X, Y$, and $Z$ was collected for each interval. Results are presented in the Table 2.

Table 2. Absolute distance error and standard deviation for outdoor long-range tag readings

\section{DISTANCE}

4m 8m 12m 16m 20m 24m 28m 32m 36m 40m

\begin{tabular}{|c|c|c|c|c|c|c|c|c|c|c|c|}
\hline $\mathbf{X}$ & 0.20 & 0.36 & 0.50 & 0.60 & 0.59 & 0.69 & 0.72 & 0.74 & 0.79 & 0.86 \\
\hline $\mathbf{X s d}$ & 0.01 & 0.02 & 0.04 & 0.05 & 0.06 & 0.08 & 0.08 & 0.07 & 0.08 & 0.08 \\
\hline $\mathbf{Y}$ & 0.28 & 0.38 & 0.45 & 0.58 & 0.68 & 0.71 & 0.73 & 0.77 & 0.82 & 0.84 \\
\hline $\mathbf{Y s d}$ & 0.02 & 0.03 & 0.03 & 0.04 & 0.04 & 0.05 & 0.06 & 0.07 & 0.06 & 0.07 \\
\hline $\mathbf{Z}$ & 0.15 & 0.16 & 0.35 & 0.39 & 0.59 & 0.65 & 0.72 & 0.76 & 0.78 & 0.88 \\
\hline Zsd & 0.01 & 0.01 & 0.02 & 0.04 & 0.03 & 0.06 & 0.06 & 0.06 & 0.09 & 0.1 \\
\hline
\end{tabular}


Calculation of the position standard error for the 40.0-m distance, considered the worst case, is done using the standard deviation of absolute position error, shown on Eq. 1.

$$
\text { P.S.E }=(\text { Abs_STD_Error }) /(400 \text { samples }),
$$

The calculation results in a Position Standard Error (P.S.E) equal to 0.007 m. Using a confidence interval of $95 \%$, the calculated measurement error results

$$
C . I=(0.86) \pm(1.96 \times 0.007)=0.86 \pm 0.014 \mathrm{~m},
$$

where 0.86 is the mean of absolute position error for the data.

The confidence interval is between $0,86 \mathrm{~m}$ and $0,87 \mathrm{~m}$, adequate to estimate the real position of the UAV, considering that a regular GPS has $4.0-\mathrm{m}$ assurance for the same situation.

\subsection{Position Accuracy of an UAV Flight Using AR-Tag}

The next experiment comprises the evaluation of the percentage error of an entire flight. An Emild Reach RTK-GPS module [2] embedded in Bebop Drone provides a position ground truth. The drone was manually piloted to take off and reach an estimated 30.0-m height. After that, the drone executed a rectangular path, at $1.5 \mathrm{~m}$ per second velocity. A $1.0-\mathrm{m}$ size AR-Tag was placed near to the center of the path. The base station computer records The RTK and AR-Tag position data during the flight. The process was repeated for five rounds.

The Fig. 3 shows a graph of one executed fly, comparing the RTK-GPS position readings (in blue) with the AR-Tag position readings (in red).

A calculation of the mean percentage error for Horizontal and Vertical position, using data of all rounds, results in

$$
\text { Horiz_Perc_Mean_Error }=7.6 \%
$$

and,

$$
\text { Vert_Perc_Mean_Error }=9,3 \% .
$$

At $30.0-\mathrm{m}$ distance of the tag, the horizontal error is near to $2.1 \mathrm{~m}$, and the vertical error is near to $2.7 \mathrm{~m}$. The results present a gain of accuracy when compared to a regular GPS error, about $4 \mathrm{~m}$. Besides that, these results must be improved to allow a proper application to the power line inspection problem, because a large position error like that could offer a high risk of collision. The fusion of odometry data and AR Tag data is probably a good approach to improve the accuracy of the solution. Future works proposes the evaluation of these techniques. 
A. R. Cantieri et al.

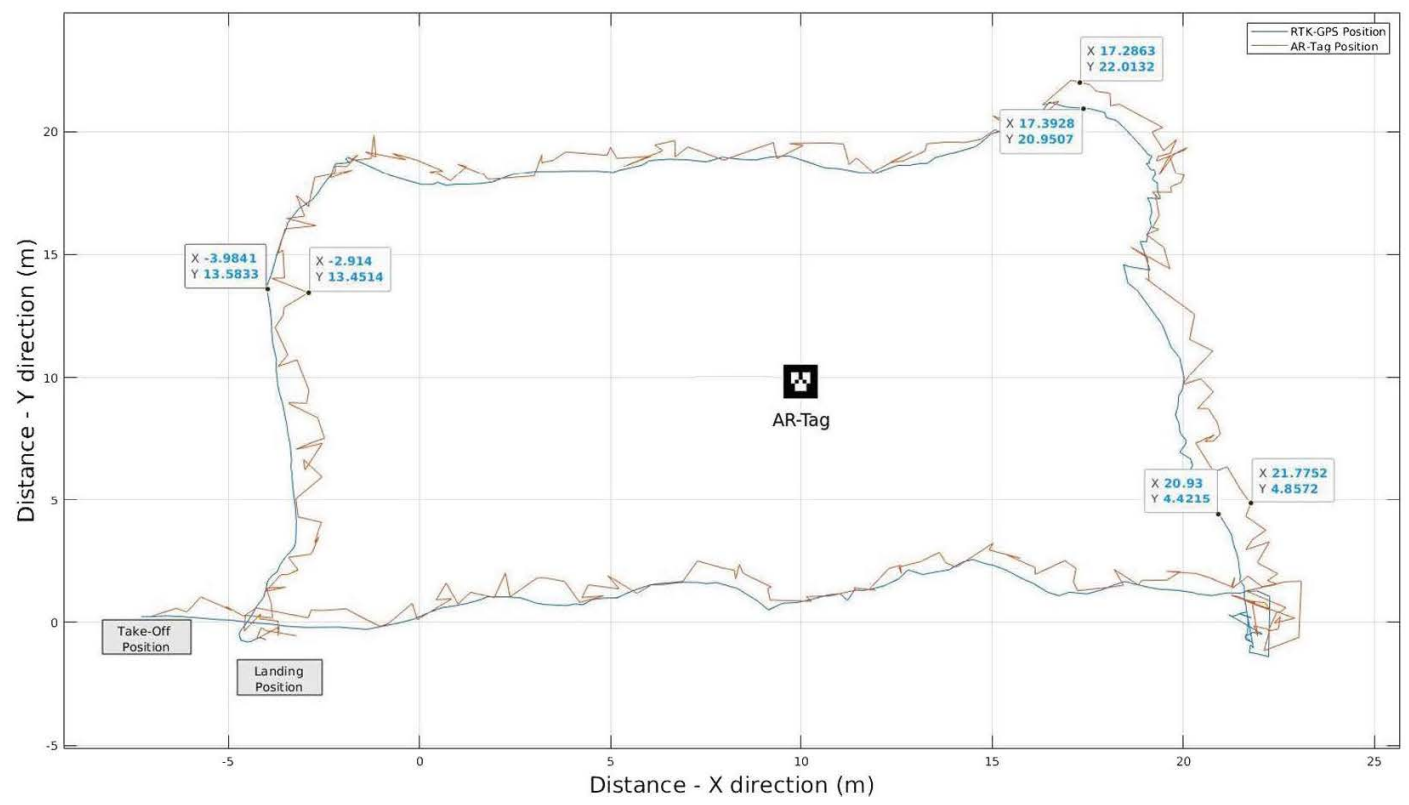

Fig. 3. Path position measured differences between RTK-GPS and AR-Tag

\subsection{Simulation Tests}

The next experiment describes the simulation of an environment with a power line tower, built to evaluate the autonomous fly of an UAV around the tower using a group of AR-Tag as position reference system. Four $1.0 \mathrm{~m}$ size AR-Tags are placed on the ground around the tower, each one presenting a different tag number to allow the differentiation between then. The system calculates the position of each tag concerning the center of the tower. Only one tag data position is used at each time by the system. The system calculates the nearest tag to the center of the UAV frame and decompresses the other tag information.

An UAV model with position and stabilization controller script receives a waypoints list from a $\mathrm{C}++$ program and execute the UAV navigation. Another $\mathrm{C}++$ program identifies the closest tag from the UAV base and calculates the UAV position using the data published on the ar_pose_marker node. This data is published on a ROS topic and feeds the LUA PID control algorithm.

Vision sensor aperture angle is $62^{\circ}, 512 \times 512$ pixel resolution. The lowest height of fly defined in this experiment is equal to $10 \mathrm{~m}$. At this height, the image sensor can capture at least one tag image on a $20-\mathrm{m}$ radius distance from the center of the tower. The four tags are placed at a $7-\mathrm{m}$ distance from the tower center on the diagonal direction. This radius is the range of work for the position system.

Four different waypoint missions were executed, in 5 rounds each. The routes were programmed manually, and the maxim distance between two consecutive waypoints is equal to $4 \mathrm{~m}$. Figure 4 shows an image of a mission and the trace $3 \mathrm{D}$ tag measured position versus the real position. 

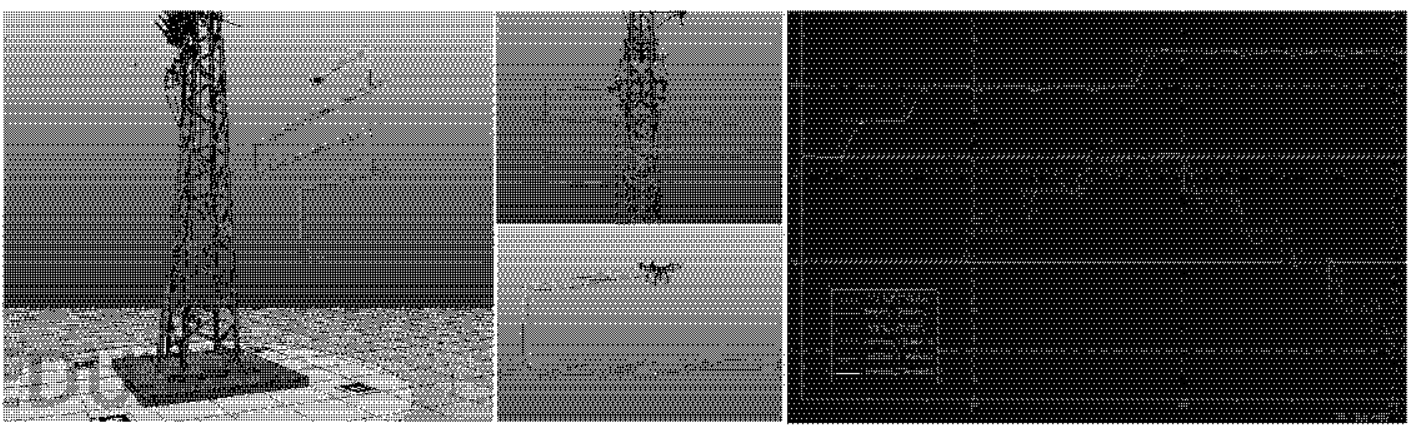

Fig. 4. Graphic visualization of UAV position error

The control algorithm was able to accomplish correctly 17 of 20 missions. In three missions, the UAV lost the position reference, and the control algorithm fails. These situations occurred when the UAV lost partial vision of the reference tag because of position swing when a new displacement begins. This swing occurs because of the simplicity of PID control algorithm, which allows high-velocity commands when the UAV is far from the waypoint.

A possible way to correct this problem is to use a robust control algorithm, as a fuzzy logic-based for example. This kind of algorithm provides soft displacements to the UAV even when the waypoints are far one from another. Application of a Kalman Filter also could bring more robustness to the position inference in this case. Future works search this solutions approach.

\section{Results Analysis and Conclusions}

This paper presents a complementary UAV position solution based on using Ar Tag visual information. The solution offers a second level position data, to be applied when traditional systems, like GPS, presents lost of accuracy. The solution uses a regular camera and processing hardware to provide the position data, offering a cheap and easy to use solution to the proposed problem.

Results obtained on the experiments and simulations show that the use of the proposed solution in real-world situation is technically viable. Simulation shows that the solution allows the creation of a trustworthy positioning system to develop UAV inspection systems, in particular on the power line inspections problem. Some obtained data presents a measurement error that could be minimized using additional data processing techniques and robust algorithms application. The use of high-resolution image to process the tag's visual information, and artificial lighting are also a proper choice to improve the system accuracy. This research is a work in progress, and next steps include new rounds of experiments to evaluate additional tools and solutions, to improve the results obtained this far. 


\section{References}

1. Bian, J., Hui, X., Zhao, X., Tan, M.: A point-line-based SLAM framework for UAV close proximity transmission tower inspection. In: 2018 IEEE International Conference on Robotics and Biomimetics, ROBIO 2018, pp. 1016-1021 (2019). https://doi.org/10.1109/ROBIO.2018.8664716

2. Emild: EMILD REACH DOCUMENTATION WEB SITE (2019). https://docs. emlid.com/reach/\#discussion

3. V.T.R.C. of Finland: Augmented Reality/3D Tracking (2017). http://virtual.vtt. fi/virtual/proj2/multimedia/

4. Hinas, A., Roberts, J.M., Gonzalez, F.: Vision-based target finding and inspection of a ground target using a multirotor UAV system. Sensors 17(12) (2017). https:// doi.org/10.3390/s17122929

5. April Robotics Laboratory: AprilTags Visual Fiducial System (2018). https://april. eecs.umich.edu/software/apriltag

6. Larrauri, J.I., Sorrosal, G., Gonzalez, M.: Automatic system for overhead power line inspection using an unmanned aerial vehicle RELIFO project. In: 2013 International Conference on Unmanned Aircraft Systems (ICUAS), pp. 244-252, May 2013. https://doi.org/10.1109/ICUAS.2013.6564696. http://ieeexplore.ieee. org/lpdocs/epic03/wrapper.htm?arnumber $=6564696$

7. Luque-Vega, L.F., Castillo-Toledo, B., Loukianov, A., Gonzalez-Jimenez, L.E.: Power line inspection via an unmanned aerial system based on the quadrotor helicopter. In: Proceedings of the Mediterranean Electrotechnical Conference - MELECON, pp. 393-397 (2014). https://doi.org/10.1109/MELCON.2014.6820566

8. Monajjemi, M.: Bebop Autonomy - ROS Driver for Parrot Bebop Drone (2018). https://bebop-autonomy.readthedocs.io/en/latest/

9. Muskardin, T., Balmer, G., Persson, L., Wlach, S., Laiacker, M., Ollero, A., Kondak, K.: A novel landing system to increase payload capacity and operational availability of high altitude long endurance UAVs. J. Intell. Robot. Syst.: Theory Appl. 88(2-4), 597-618 (2017). https://doi.org/10.1007/s10846-017-0475-z

10. Rangel, R.K., Kienitz, K.H., Brandão, M.P.: Development of a multi-purpose portable electrical UAV system, fixed \& rotative wing. In: 2011 IEEE Aerospace Conference (2011)

11. ROS.org: ROS (2019). http://www.ros.org

12. USA: US Department of Defense: Global Positioning System Standard Positioning Service, pp. 1 - 160, September 2008. http://www.Gps.Gov, http://www.gps.gov/ technical/ps/2008-SPS-performance-standard.pdf

13. Zimmermann, F., Eling, C., Klingbeil, L., Kuhlmann, H.: Precise positioning of UAVs - dealing with challenging RTK-GPS measurement conditions during automated UAV flights. ISPRS Ann. Photogramm. Remote Sens. Spatial Inf. Sci. 4(2W3), 95-102 (2017). https://doi.org/10.5194/isprs-annals-IV-2-W3-95-2017 


\section{Source details}

\section{Advances in Intelligent Systems and Computing}

Formerly known as: Advances in Intelligent and Soft Computing

Scopus coverage years: from 2005 to 2006, 2008, 2010, from 2012 to Present

Publisher: Springer Nature

ISSN: 2194-5357

Subject area: Computer Science: General Computer Science

Engineering: Control and Systems Engineering

View all documents >

Set document alert

四 Save to source list Sarvissis 04

CiteScore 2018

0.54

(1)

Add CiteScore to your site

SJR 2018

0.174

(1)

SNIP 2018

0.434

CiteScore CiteScore rank \& trend CiteScore presets Scopus content coverage

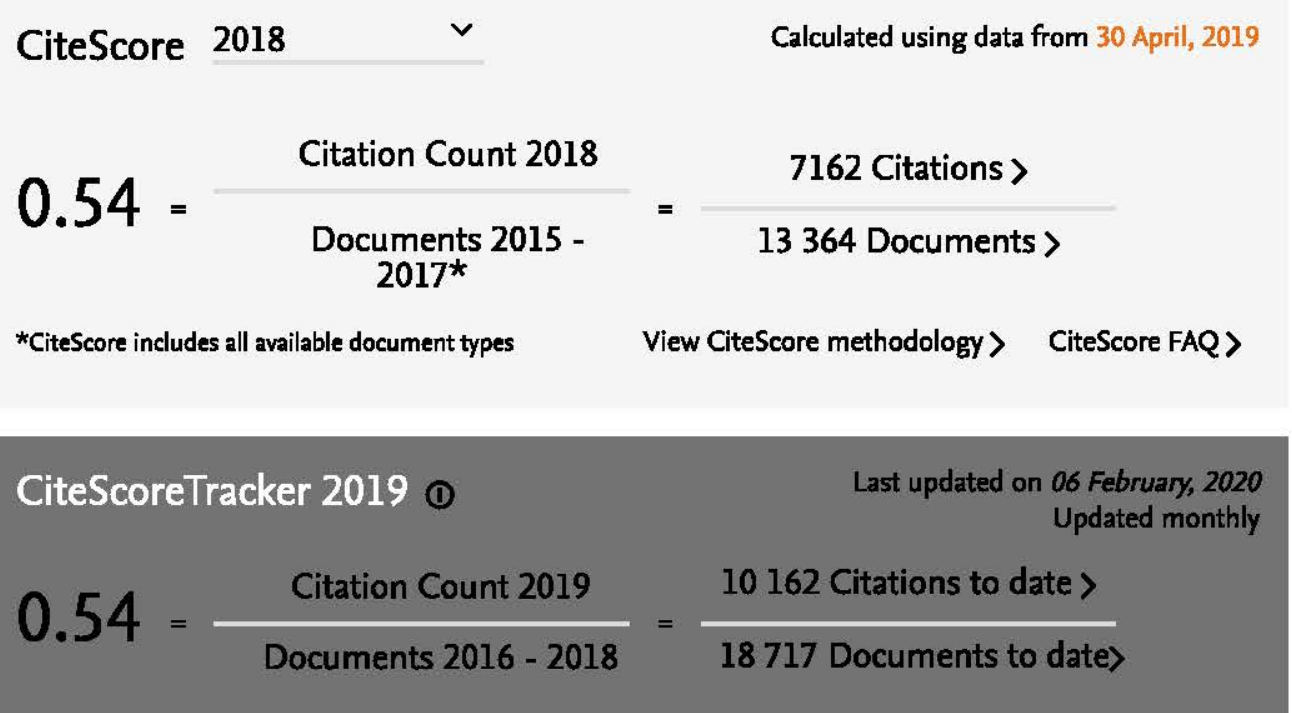

Metrics displaying this icon are compiled according to Snowball Metrics $\pi$, a collaboration between industry and academia.

\section{CiteScore rank (1)}

\begin{tabular}{|c|c|c|}
\hline Category & Rank & Percentile \\
\hline \multicolumn{3}{|l|}{ Computer Science } \\
\hline $\begin{array}{l}\text { L General } \\
\text { Computer } \\
\text { Science }\end{array}$ & \#156/206 & 24th \\
\hline \multicolumn{3}{|l|}{ Engineering } \\
\hline $\begin{array}{l}\text { L Control and } \\
\text { Systems } \\
\text { Engineering }\end{array}$ & \#191/233 & 18th \\
\hline
\end{tabular}

View CiteScore trends > 

About Scopus
Language
日本語に切り替える
切换到简体中文
切換到繁體中文
Русский язык

\section{Customer Service}
Help
Contact us
Scopus blog
Scopus API
Privacy matters
Terms and conditions $\pi \quad$ Privacy policy $\pi$
Copyright (C) Elsevier B.V ז. All rights reserved. Scopus is a registered trademark of Elsevier B.V.
We use cookies to help provide and enhance our service and tailor content. By continuing, you agree to the use of cookies. 